Pacific Journal of Mathematics

FOURIER COEFFICIENTS OF NONHOLOMORPHIC
MODULAR FORMS AND SUMS OF KLOOSTERMAN SUMS 


\section{FOURIER COEFFICIENTS \\ OF NON-HOLOMORPHIC MODULAR FORMS AND SUMS OF KLOOSTERMAN SUMS}

\section{Ka-LAM KUEH}

This paper studies Fourier coefficients of non-holomorphic modular forms and sums of Kloosterman sums.

1. Introduction. Put $\Gamma=\operatorname{PSL}(2, Z)$ and $H^{+}=\{x+i y \mid y>0\}$. Consider the Hilbert space $\mathfrak{L}^{2}\left(H^{+} / \Gamma\right)$ of function $u(z)$ satisfying:

$$
u(\gamma z)=u(z) \quad(\gamma \in \Gamma)
$$

and

$$
\langle u, u\rangle=\iint_{H^{+} / \Gamma}|u(z)|^{2} \frac{d x d y}{y^{2}}<+\infty .
$$

Consider the Laplacian $\Delta$ on $\mathfrak{L}^{2}\left(H^{+} / \Gamma\right)$ :

$$
\Delta=-y^{2}\left(\frac{\partial^{2}}{\partial x^{2}}+\frac{\partial^{2}}{\partial y^{2}}\right) \text {. }
$$

A function $u(z)$ in $\mathfrak{L}^{2}\left(H^{+} / \Gamma\right)$ is called a cusp form if the constant term in the Fourier expansion of $u(z)$ vanishes. It is known that the Laplacian $\Delta$ has a complete discrete spectral decomposition on the subspace of cusp forms. The Maass wave forms $u_{j}(z)$ defined by

$$
\Delta u_{j}(z)=\lambda_{j} u_{j}(z), \quad\left\langle u_{j}, u_{j}\right\rangle=1,
$$

where $\lambda_{1} \leq \lambda_{1} \leq \lambda_{3} \leq \cdots$ are the discrete eigenvalues of $\Delta$, constitute an orthonormal basis for the subspace of cusp forms. Note that $\lambda_{1}>$ $\frac{3}{2} \pi^{2}$. From (1) we have the Fourier expansion:

$$
u_{j}(z)=\sqrt{y} \sum_{n \neq 0} \rho_{j}(n) K_{i k_{j}}(2 \pi \mid n) e(n x), \quad e(\theta)=e^{2 \pi i \theta}
$$

where $\lambda_{j}=\frac{1}{4}+k_{j}^{2}$ and $K_{i k_{j}}(\cdot)$ is the Whittaker function. We have

$$
\#\left\{k_{j}|| k_{j} \mid \leq X\right\}=\frac{1}{12} X^{2}+c X \log X+O(X)
$$

where $c$ is a constant; cf. Venkov [7]. 
An important problem in the theory of non-holomorphic modular form is to estimate the Fourier coefficients $\rho_{j}(n)$. The RamanujanPeterson conjecture states that for large $|n|$

$$
\rho_{j}(n) \underset{\varepsilon, j}{\ll}|n|^{\varepsilon} \quad(\varepsilon>0) .
$$

A method to study the Fourier coefficients $\rho_{j}(n)$ of $u_{j}(z)$ is the non-holomorphic Poincaré series introduced by Selberg [5]:

$$
P_{m}(z, s)=\sum_{\gamma \in \Gamma / \Gamma_{\infty}}(\operatorname{Im} \gamma z)^{s} e(m \gamma z) \quad(\operatorname{Re} s>1),
$$

where $m \geq 1$ is an integer and $\Gamma_{\infty}$ is the subgroup of translations. The Poincaré series belongs to $\mathfrak{L}^{2}\left(H^{+} / \Gamma\right)$, and its inner product against a function $u(z) \in \mathfrak{L}^{2}\left(H^{+} / \Gamma\right)$ gives the $m$ th Fourier coefficient of $u(z)$. Selberg [5] obtained the meromorphic continuation of $P_{m}(z, s)$ to the entire complex $s$-plane. By considering the inner product of two Poincaré series, Kuznietsov [4] developed summation formulas connecting the Fourier coefficients $\rho_{j}(n)$ and the Kloosterman sum

$$
S(m, n ; c)=\sum_{\substack{d=1 \\ a d \equiv 1(\bmod c)}}^{c} e\left(\frac{a m+d n}{c}\right) .
$$

One of the summation formulas useful to us is equation (9) below. By using the summation formulas, Kuznietsov [4] proved that

$$
\sum_{0<k_{j}<X} \frac{\left|\rho_{j}(n)\right|^{2}}{c h \pi k_{j}}=\frac{1}{\pi^{2}} X^{2}+O\left(X \log X+X n^{\varepsilon}+n^{1 / 2+\varepsilon}\right),
$$

and

$$
\sum_{c<T} \frac{S(m, n ; c)}{c} \underset{m, n}{\ll} T^{1 / 6} \log ^{1 / 3} T .
$$

The Weil estimate gives

$$
|S(m, n ; c)| \leq(m, n, c)^{1 / 2} d(c) c^{1 / 2},
$$

which yields a trivial bound $O\left(T^{1 / 2+\varepsilon}\right)$ for the sum in (6).

The Linnik-Selberg conjecture states that

$$
\sum_{c \leq T} \frac{S(m, n ; c)}{c} \ll_{\varepsilon} T^{\varepsilon} \quad\left(T>(m, n)^{1 / 2}, \varepsilon>0\right) .
$$


To deal with the estimate of $\rho_{j}(n)$, Selberg [5] introduced the above conjecture.

Another method to study the sum of Kloosterman sum in (6) is by the Kloosterman zeta function introduced by Selberg [5]:

$$
Z_{m, n}(s)=\sum_{c=1}^{\infty} \frac{S(m, n ; c)}{c^{2 s}} \quad\left(\operatorname{Re} s>\frac{3}{4}\right) .
$$

Selberg [5] obtained the meromorphic continuation of $Z_{m, n}(s)$ to the entire complex plane. A useful characterization of $Z_{m, n}(s)$ may be found in (7.26) of Kuznietsov [4].

Goldfeld and Sarnak [3] have given a very simple proof of the bound $O\left(T^{1 / 6+\varepsilon}\right)$ for the sum in (6) by proving a good bound on $Z_{m, n}(s)$ in the critical strip.

Equation (5) means that on the average $\left|\rho_{j}(n)\right|^{2} / c h \pi k_{j}$ is bounded with respect to the indices $k_{j}$ from 0 to $X$. In this paper, we will show the following:

THEOREM 1. We have for $n^{1+\varepsilon} \ll t \quad(\varepsilon>0)$,

$$
\sum_{\left|k_{j}-t\right|<1} \frac{\left|\rho_{j}(n)\right|^{2}}{c h \pi k_{j}} \ll t \quad(t \rightarrow+\infty) .
$$

Theorem 1 means that on the average $\left|\rho_{j}(n)\right|^{2} / c h \pi k_{j}$ is bounded with respect to $k_{j}$ in short interval.

With Theorem 1, we will show furthermore

THEOREM 2. For any $f(t) \rightarrow+\infty$ and $f(t)=o(t)$ as $t \rightarrow+\infty$, and $n^{1+\varepsilon} \ll t \quad(\varepsilon>0)$, we have

$$
\sum_{\left|k_{j}-t\right|<f(t)} \frac{\left|\rho_{j}(n)\right|^{2}}{c h \pi k_{j}} \sim \frac{4}{\pi^{2}} t f(t) \quad(t \rightarrow+\infty)
$$

and

THEOREM 3. For $Y \geq 10$, we have

$$
\int_{Y}^{e Y}\left(\sum_{c \leq x} \frac{S(m, n ; c)}{d}\right)^{2} \frac{d x}{x} \underset{m, n}{\ll} \log Y .
$$


It may be interesting to note that we get as a by-product of the proof of Theorem 2 the following:

Theorem 4. For any $\sigma \in \mathbb{C}$, we have

$$
\int_{-\infty}^{\infty} \Gamma\left(\sigma-\frac{1}{2}-i r\right) \Gamma\left(\sigma-\frac{1}{2}+i r\right) d r=\pi 2^{2-2 \sigma} \Gamma(2 \sigma-1) .
$$

Theorem 4 would follow immediately from the proof of Theorem 2 . In view of (3), it may be interesting to compare Kuznietsov's estimate (5) with Theorems 1 and 2. Theorem 3 means that the sum in (6) is "very small" for almost all $x$ and for most of the time better than the Linnik-Selberg conjecture. More precisely, for $Y \geq 10$ and $f(x) \nearrow$ $\infty$, let $M_{Y} \subset[Y, e Y]$ such that

$$
\left|\sum_{c<x} \frac{S(m, n ; c)}{c}\right| \geq f(x) \log ^{1 / 2} x \quad\left(x \in M_{Y}\right) .
$$

Then Theorem 3 shows that the Lebesgue measure of $M_{Y}$ is $O\left(f(Y)^{-2} Y\right)$.

By putting $\sigma=\frac{3}{4}+1 / \log n$ in Lemma 1 , Theorem 1 follows immediately. We prove Theorem 3 by establishing Lemma 2, which is analogous to the explicit formula in the theory of prime number, and by using Gallagher's mean-value inequality for exponential sum which is Lemma 3. The method imitates an idea of Gallagher [2].

2. Lemmas. The proof of Lemma 1 is based on the following equation (9) which follows by putting $s_{1}=\sigma+i t$ and $s_{2}=\sigma-i t$ in the lemmas in $\S 4.1$ and $\S 4.4$ of Kuznietsov [4].

Proposition. For $s=\sigma+i t, \frac{3}{4}<\sigma<\frac{5}{4}$, and any integer $n \geq 1$, we have

$$
\begin{aligned}
& \pi\left\{\sum_{j=1}^{\infty}\left|\rho_{j}(n)\right|^{2} \Lambda\left(s ; k_{j}\right)\right. \\
&\left.\quad+\frac{1}{\pi} \int_{-\infty}^{\infty}\left|\sigma_{2 i r}(n)\right|^{2} \Lambda(s ; r) \frac{c h \pi r}{|\zeta(1+2 i r)|^{2}} d r\right\} \\
&= \Gamma(2 \sigma-1)+(4 \pi n)^{2 \sigma-1} \\
& \times\left\{\frac{2^{3-2 \sigma}}{i s h 2 \pi t} \sum_{c=1}^{\infty} \frac{S(n, n ; c)}{c^{2 \sigma}} \Phi\left(s, \frac{4 \pi n}{c}\right)\right\},
\end{aligned}
$$


where

$$
\Lambda(s ; r)=\frac{\left|\Gamma\left(s-\frac{1}{2}+i r\right) \Gamma\left(s-\frac{1}{2}-i r\right)\right|^{2}}{|\Gamma(s)|^{2}}, \quad \sigma_{2 i r}(n)=\sum_{d \mid n} d^{2 i r}
$$

and for $x>0$

$$
\begin{aligned}
\Phi(s, x)=-\pi \int_{1}^{\infty}\left(u-\frac{1}{u}\right)^{2 \sigma-2}\{( & \sin \pi s) J_{2 i t}(x u) \\
& \left.+(\sin \pi \bar{s}) J_{-2 i t}(x u)\right\} \frac{d u}{u}
\end{aligned}
$$

and $J_{2 i t}(u)$ is the Bessel function.

We need the following estimate for the Bessel function:

$$
J_{i t}(u) \ll e^{\pi t / 2}\left(t^{2}+u^{2}\right)^{-1 / 4} \quad(t \in \mathbb{R})
$$

uniformly in $u>0$ for $|t| \rightarrow+\infty$.

LEMMA 1. We have for $\frac{3}{4}<\sigma<\frac{5}{6}$

$$
\sum_{\left|t-k_{j}\right|<1} \frac{\left|\rho_{j}(n)\right|^{2}}{c h \pi k_{j}} \ll t+\sqrt{t} n^{2 \sigma-1}\left(\sigma-\frac{3}{4}\right)^{-2} \quad(t \rightarrow+\infty) .
$$

Proof. We take $\frac{3}{4}<\sigma<\frac{5}{6}$ in the Proposition. With the bound in (11), we see from (10) that

$$
\begin{aligned}
& \Phi(s, x) \ll e^{2 \pi t} \int_{1}^{\infty}\left(u-\frac{1}{u}\right)^{2 \sigma-2}\left(t^{2}+x^{2} u^{2}\right)^{-1 / 4} \frac{d u}{u} \\
& \quad\left(x=\frac{4 \pi n}{c}\right) \\
& \ll t^{-1 / 2} e^{2 \pi t} \int_{1}^{\infty}\left(u-\frac{1}{u}\right)^{2 \sigma-2}\left(1+\left(\frac{x}{t}\right)^{2} u^{2}\right)^{-1 / 4} \frac{d u}{u} \\
& \ll t^{-1 / 2} e^{2 \pi t}, \quad \text { since } \frac{3}{4}<\sigma<\frac{5}{6} .
\end{aligned}
$$

On considering Weil's bound for $S(m, n ; c)$ and (13), the second term on the right-hand side of (9) is then

$$
\ll t^{-1 / 2} n^{2 \sigma-1}\left(\sigma-\frac{3}{4}\right)^{-2}
$$


On the other hand, the integral in (9) is non-negative, and the series in (9) is

$$
\begin{aligned}
& \geq \sum_{\left|k_{j}-t\right|<1}\left|\rho_{j}(n)\right|^{2} \frac{\left|\Gamma\left(s-\frac{1}{2}+i k_{j}\right) \Gamma\left(s-\frac{1}{2}-i k_{j}\right)\right|^{2}}{|\Gamma(s)|^{2}} \\
& \gg \frac{1}{t} \sum_{\left|k_{j}-t\right|<1} \frac{\left|\rho_{j}(n)\right|^{2}}{c h \pi k_{j}},
\end{aligned}
$$

since $\Gamma(s)=\sqrt{2 \pi} e^{-(\pi / 2)|t|} t^{\sigma-1 / 2}\left(1+O\left(|t|^{-1}\right)\right)$, and $\left|\Gamma\left(s-\frac{1}{2}-i k_{j}\right)\right| \gg 1$ for $\left|t-k_{j}\right|<1$.

This proves Lemma 1.

LEMMA 2. We have for $T<\frac{1}{2} x$

$$
\sum_{c \leq x} \frac{S(m, n ; c)}{c}=\sum_{\left|k_{j}\right|<T} \frac{\rho_{j}(n) \overline{\rho_{j}(m)}}{c h \pi k_{j}} \frac{\Gamma\left(2 i k_{j}\right)}{2 i k_{j}} x^{2 i k_{j}}+O\left(\frac{x^{1 / 2} \log ^{2} x}{T}\right)
$$

the implicit constant here depends on $m, n$.

Before proceeding with the proof of Lemma 2, we need several analytic properties of $Z_{m, n}(s)$. On the half plane $\operatorname{Re} s>0$, the poles of $Z_{m, n}(s)$ are located at $s=\frac{1}{2}+i k_{j}$, and as $t \rightarrow \infty$

$$
Z_{m, n}(s) \underset{m, n}{\ll} \frac{|s|^{1 / 2}}{\left|\sigma-\frac{1}{2}\right|} \quad\left(s=\sigma+i t, \sigma \neq \frac{1}{2}\right) .
$$

Estimate (15) is obvious by using the result and the same method as in the proof of Theorem 1 of Goldfeld and Sarnak [3]. On the other hand, by the Lemma of $\S 7.3$ of Kuznietsov [4], we have the representation for $Z_{m, n}(s) \quad(s \in \mathbb{C})$ :

$$
\begin{aligned}
(2 \pi \sqrt{m n})^{2 s-1} Z_{m, n}(s) \\
=\sum_{j=1}^{\infty} \frac{\rho_{j}(n) \overline{\rho_{j}(m)}}{c h \pi k_{j}} h\left(k_{j}, s\right)-\frac{\delta_{m, n}}{2 \pi} \frac{\Gamma(s)}{\Gamma(1-s)} \\
\quad+\sum_{l=0}^{\infty} p_{m, n}(l) \frac{\Gamma(s+l)}{\Gamma(2-s+l)}+L_{m, n}(s)
\end{aligned}
$$


where $L_{m, n}(s)$ denotes the analytic continuation of the function which is defined in the half plane $\operatorname{Re} s>\frac{1}{2}$ by the integral

$$
L_{m, n}(s)=\frac{1}{\pi} \int_{-\infty}^{\infty}\left(\frac{n}{m}\right)^{i t} \sigma_{2 i r}(n) \sigma_{-2 i r}(m) \frac{h(r, s)}{\zeta(1+2 i r) \zeta(1-2 i r)} d r,
$$

and

$$
h(r, s)=\frac{1}{2} \sin (\pi s) \Gamma\left(s-\frac{1}{2}+i r\right) \Gamma\left(s-\frac{1}{2}-i r\right),
$$

and

$$
p_{m, n}(l)=(2 l+1) \sum_{c=1}^{\infty} \frac{S(m, n ; c)}{c} J_{2 l+1}\left(\frac{4 \pi \sqrt{m n}}{c}\right) .
$$

By (16), we see that

$$
\operatorname{Re}_{s=1 / 2+i k_{j}} Z_{m, n}(s)=\frac{(2 \pi \sqrt{m n})^{-2 i k_{j}}}{2} \Gamma\left(2 i k_{j}\right) \rho_{j}(n) \overline{\rho_{j}(m)} .
$$

Consider $s=\sigma+i t$ with

$$
\left|\sigma-\frac{1}{2}\right| \leq \frac{\delta}{\log (|t|+2)}
$$

for a small $\delta>0$. Deforming suitably the integral path in the integral of $L_{m, n}(s)$, we have for $s$ satisfying (18)

$$
L_{m, n}(s)=O\left(\log ^{2}|t|\right)
$$

since $\zeta(x+i y) \neq 0$ and $\zeta(x+i y) \ll \log (|y|+2)$ in the region $x>$ $1-(c / \log (|y|+2))(c>0)$.

Also for $s$ satisfying (18), we have

$$
\frac{\Gamma(s)}{\Gamma(1-s)} \ll 1
$$

and

$$
\sum_{l=0}^{\infty} p_{m, n}(l) \frac{\Gamma(s+l)}{\Gamma(2-s+l)} \ll m n .
$$

By using the estimate on Bessel function

$$
\left|J_{k}(y)\right| \leq \min \left(1, \frac{(y / 2)^{k}}{(k-1) !}\right)
$$


we prove $(21)$ as follows: note first that $(2 l+1) \Gamma(s+l) / \Gamma(2-s+l) \ll$ 1. Thus

$$
\begin{aligned}
\sum_{l=0}^{\infty} p_{m, n}(l) \frac{\Gamma(s+l)}{\Gamma(2-s+l)} & \\
\ll & \sum_{l=0}^{\infty} \sum_{1 \leq c \leq 20 \pi \sqrt{m n}} \frac{|S(m, n ; c)|}{c}\left|J_{2 l+1}\left(\frac{4 \pi \sqrt{m n}}{c}\right)\right| \\
& +\sum_{l=0}^{\infty} \sum_{c>20 \pi \sqrt{m n}} \frac{|S(m, n ; c)|}{c}\left|J_{2 l+1}\left(\frac{4 \pi \sqrt{m n}}{c}\right)\right| \\
\ll & \sum_{0 \leq l \leq 20 \pi \sqrt{m n}} \sum_{1 \leq c \leq 20 \pi \sqrt{m n}} \frac{|S(m, n ; c)|}{c}\left|J_{2 l+1}\left(\frac{4 \pi \sqrt{m n}}{c}\right)\right| \\
& +\sum_{1 \leq c \leq 20 \pi \sqrt{m n}} \frac{|S(m, n ; c)|}{c}\left|J_{2 l+1}\left(\frac{4 \pi \sqrt{m n}}{c}\right)\right| \\
& +\sum_{l>20 \pi \sqrt{m n}} \frac{|S(m, n ; c)|}{c} \sum_{l=0}^{\infty}\left|J_{2 l+1}\left(\frac{4 \pi \sqrt{m n}}{c}\right)\right| \\
\ll & +\sum_{1 \leq c \leq 20 \pi \sqrt{m n}} \frac{|S(m, n ; c)|}{c} \sum_{l>20 \pi \sqrt{m n}}\left(\frac{2 \pi \sqrt{m n}}{c}\right)^{2 l+1} \frac{1}{(2 l) !}
\end{aligned}
$$$$
+\sum_{c>20 \pi \sqrt{m n}} \frac{|S(m, n ; c)|}{c} \sum_{l=0}^{\infty}\left(\frac{2 \pi \sqrt{m n}}{c}\right)^{2 l+1} \frac{1}{(2 l !)}
$$

$$
\begin{aligned}
\ll m n & +\sum_{l \leq c \leq 20 \pi \sqrt{m n}} \frac{|S(m, n ; c)|}{c} \\
& +\sum_{c>20 \pi \sqrt{m n}} \frac{|S(m, n ; c)|}{c} \times \frac{2 \pi \sqrt{m n}}{c}
\end{aligned}
$$

$\ll m n$.

This proves (21). Estimate (21) is obviously not the best, but we are satisfied with this presently.

Also by using Theorem 1 and (5), we have that

$$
\sum_{\left|k_{j}-t\right|>1} \frac{\rho_{j}(n) \overline{\rho_{j}(m)}}{c h \pi k_{j}} h\left(k_{j}, x\right)=O(|t|)
$$

for $s$ satisfying (18) and $\max \left\{m^{1+\varepsilon}, n^{1+\varepsilon}\right\} \ll|t|$. Thus by (19), (20), 
(21) and (22), equation (16) becomes

$$
Z_{m, n}(s)=(2 \pi \sqrt{m n})^{1-2 s} \sum_{\left|k_{j}-t\right|<1} \frac{\rho_{j}(n) \overline{\rho_{j}(m)}}{c h \pi k_{j}} h\left(k_{j}, s\right)+O(|t|)
$$

for $s$ satisfying (18) and $m n \ll|t|$ and $\max \left\{m^{1+\varepsilon}, n^{1+\varepsilon}\right\} \ll|t|$.

We are now in a position to prove Lemma 2.

Proof of Lemma 2. Choose $0<\varepsilon \leq \delta / \log (|t|+2)$ for small $\delta>0$. By (15) and the Lindelöf-Phragmen principle it follows that

$$
\left|Z_{m, n}(s)\right| \underset{m, n}{\ll} \frac{|t|^{3 / 2-2 \sigma+2 \varepsilon}}{\varepsilon^{2}}
$$

for $\frac{1}{2}+\varepsilon \leq \sigma \leq \frac{3}{4}+\varepsilon$, since $Z_{m, n}\left(\frac{3}{4}+\varepsilon\right) \underset{m, n}{\ll} \varepsilon^{-2}$ by (8); and obviously

$$
\left|Z_{m, n}(s)\right| \underset{m, n}{\ll} \frac{|t|^{1 / 2}}{\frac{1}{2}-\sigma}
$$

for $\frac{1}{10} \leq \sigma \leq \frac{1}{2}-\varepsilon$.

Consider the integral

$$
I(T)=\frac{1}{2 \pi i} \int_{\eta-i T}^{\eta+i T} Z_{m, n}(s) \frac{x^{2 s-1}}{2 s-1} d s \quad\left(\eta=\frac{3}{4}+\varepsilon\right)
$$

with $T>0$ not an ordinate of a pole of $Z_{m, n}(s)$. Now by Lemma 3.12 of Titchmarsh [6], we get

$$
\begin{aligned}
\sum_{c \leq x} \frac{S(m, n ; c)}{c}= & \frac{1}{2 \pi i} \int_{\eta-i T}^{\eta+i T} Z_{m, n}(s) \frac{x^{2 s-1}}{2 s-1} d s \\
& +O_{m, n}\left(\frac{x^{\eta}}{T \varepsilon^{2}}\right) .
\end{aligned}
$$

Computations of residues yield

$$
\begin{aligned}
I(T)= & \sum_{\left|k_{j}\right|<T} \xi_{j} \frac{x^{2 i k_{j}}}{2 i k_{j}}+\frac{1}{2 \pi i} \int_{1 / 10-i T}^{1 / 10+i T} Z_{m, n}(s) \frac{x^{2 s-1}}{2 s-1} d s \\
& +\frac{1}{2 \pi i} \int_{1 / 10 \pm i T}^{\eta \pm i T} Z_{m, n} \frac{x^{2 s-1}}{2 s-1} d s
\end{aligned}
$$

where $\xi_{j}$ is the residue of $Z_{m, n}(s)$ at $s=\frac{1}{2}+i k_{j}$. Using (17), we 
see that

$$
\xi_{j} \ll \frac{\left|\rho_{j}(n) \rho_{j}(m)\right|}{c h \pi k_{j}}\left|k_{j}\right|^{-1 / 2} .
$$

Now we estimate the integrals in (28). By (25), we have first

$$
\int_{1 / 10-i T}^{1 / 10+i T} Z_{m, n}(s) \frac{x^{2 s-1}}{2 s-1} d s \underset{m, n}{\ll} x^{-4 / 5} T^{1 / 2}
$$

and

$$
\int_{1 / 10 \pm i T}^{1 / 2-\varepsilon \pm i T} Z_{m, n}(s) \frac{x^{2 s-1}}{2 s-1} d s \underset{m, n}{\ll} \frac{x^{-2 \varepsilon} T^{-1 / 2}}{\varepsilon} .
$$

By (24), we have

$$
\int_{1 / 2+\varepsilon \pm i T}^{\eta \pm i T} Z_{m, n}(s) \frac{x^{2 s-1}}{2 s-1} d s \underset{m, n}{\ll} \frac{x^{1 / 2+\varepsilon}}{\varepsilon^{2}|t|} \frac{1}{\left|\log \frac{T}{x}\right|}
$$

for $\left|\log \frac{T}{x}\right| \gg 1$.

Finally, by (23) we have

$$
\begin{aligned}
\int_{1 / 2-\varepsilon \pm i T}^{1 / 2+\varepsilon \pm i T} Z_{m, n}(s) \frac{x^{2 s-1}}{2 s-1} d s \\
=\sum_{|k, \mp T|<1} \frac{\rho_{j}(n) \overline{\rho_{j}(m)}}{c h \pi k_{j}} \\
\quad \cdot \int_{1 / 2-\varepsilon \pm i T}^{1 / 2+\varepsilon \pm i T}(2 \pi \sqrt{m n})^{1-2 s} h\left(k_{j}, s\right) \frac{x^{2 s-1}}{2 s-1} d s+O_{m, n}(\varepsilon) .
\end{aligned}
$$

Noting that $|\Gamma(s)| \gg|s|^{-1}$ for $\varepsilon \ll|s| \ll 1$ and by suitably deforming the integral path on the right-hand side of (33) to an upper or lower semi-circle according as $\frac{1}{2}+i k_{j}$ stays below or above the integral path, we get

$$
\int_{1 / 2-\varepsilon \pm i T}^{1 / 2+\varepsilon \pm i T}(2 \pi \sqrt{m n})^{1-2 s} h\left(j_{k}, s\right) \frac{x^{2 s-1}}{2 s-1} d s \underset{m, n}{\ll} T^{-3 / 2}
$$

since $\left|k_{j} \mp T\right|<1$, so the right-hand side of (33) is

$$
\begin{aligned}
& \underset{m, n}{\ll} \sum_{\left|k_{j} \mp T\right|<1} \frac{\left|\rho_{j}(n) \rho_{j}(m)\right|}{c h \pi k_{j}} T^{-3 / 2}+\varepsilon \\
& \ll T^{-1 / 2}+\varepsilon,
\end{aligned}
$$

by Theorem 1 . 
Putting (30), (31), (32), and (34) together, equation (28) becomes, for $\log \left|\frac{T}{x}\right| \gg 1$ and $\varepsilon=\delta \log ^{-1} T$,

$$
I(T)=\sum_{\left|k_{j}\right|<T} \xi_{j} \frac{x^{2 i k_{j}}}{2 i k_{j}}+O_{m, n}\left(\frac{x^{1 / 2} \log ^{2} x T}{T}\right)
$$

for $T \leq \frac{1}{2} x$, which combined with (27) yield

$$
\sum_{c \leq x} \frac{S(m, n ; c)}{c}=\sum_{\left|k_{j}\right|<T} \xi_{j} \frac{x^{2 i k_{j}}}{2 i k_{j}}+O_{m, n}\left(\frac{x^{1 / 2} \log ^{2} x T}{T}\right) .
$$

This completes the proof of Lemma 2.

By putting $T=x^{1 / 3} \log ^{4 / 3} x$ in (36), we get $O\left(x^{1 / 6} \log ^{2 / 3} x\right)$ on the right-hand side of (36) which is slightly inferior to Kuznietsov's bound (6).

LEMMA 3. Let $A(u)=\sum_{v} c(v) e^{i v u}$ be an absolutely convergent series with complex coefficients $c(v)$ and real indices $v$. Then for $T>0$

$$
\int_{-T}^{T}\left|\sum_{v} c(v) e^{i v u}\right|^{2} d u \ll \int_{-\infty}^{\infty}\left|T \sum_{t<v<t+T^{-1}} c(v)\right|^{2} d t .
$$

Proof. This is Lemma 1 of Gallagher [1].

3. Proofs of Theorems. We prove first Theorem 2. Take $\sigma=\frac{3}{4}+$ $1 / \log n$. Then the Proposition of $\S 2$ gives

$$
\sum_{\left|k_{j}-t\right|<1} \frac{\left|\rho_{j}(n)\right|^{2}}{c h \pi k_{j}} \ll t \quad\left(n^{1+\varepsilon} \ll t\right),
$$

which is the assertion of Theorem 1 .

In view of $|\zeta(1+i r)|^{-1} \ll \log |r| \quad(|r| \rightarrow+\infty)$, a rough estimate gives

$$
\int_{-\infty}^{\infty}\left|\sigma_{2 i r}(n)\right|^{2} \Lambda(s ; r) \frac{c h \pi r}{|\zeta(1+2 i r)|^{2}} d r \ll t^{-1} \log t d^{2}(n) .
$$

We have, for $k_{j} \geq t+\sqrt{f(t)}$,

$$
\begin{aligned}
\Lambda\left(s, k_{j}\right) & =\frac{\left|\Gamma\left(s-1 / 2+i k_{j}\right) \Gamma\left(s-1 / 2-i k_{j}\right)\right|^{2}}{|\Gamma(s)|^{2}} \\
& \ll e^{\pi t} t^{1-2 \sigma} e^{-2 \pi k_{j}}\left|t+k_{j}\right|^{2 \sigma-2}\left|k_{j}-t\right|^{2 \sigma-2},
\end{aligned}
$$


and for $k_{j} \leq t-\sqrt{f(t)}$

$$
\Lambda\left(s ; k_{j}\right) \ll e^{-\pi t} t^{1-2 \sigma}\left|t+k_{j}\right|^{2 \sigma-2}\left|t-k_{j}\right|^{2 \sigma-2} .
$$

On considering (37) and (5), inequalities (39) and (40) give rise to

$$
\sum_{\left|t-k_{j}\right| \geq \sqrt{f(t)}}\left|\rho_{j}(n)\right|^{2} \Lambda\left(s ; k_{j}\right)=o(1) \quad(t \rightarrow+\infty) .
$$

Now (14) together with (38) and (41) yield, by virtue of (9),

$$
\sum_{\left|k_{j}-t\right|<\sqrt{f(t)}}\left|\rho_{j}(n)\right|^{2} \Lambda\left(s ; k_{j}\right)=\frac{1}{\pi} \Gamma(2 \sigma-1)+o(1),
$$

since $n^{1+\varepsilon} \ll t$. And also for $\left|k_{j}-t\right|<\sqrt{f(t)}$

$$
\Lambda\left(s ; k_{j}\right)=2^{2 \sigma-2} t^{-1} e^{-\pi k_{j}}\left|\Gamma\left(s-\frac{1}{2}-i k_{j}\right)\right|^{2}(1+o(1)) .
$$

Substituting this into (42), we obtain

$$
\begin{gathered}
\sum_{\left|k_{j}-t\right|<\sqrt{f(t)}}\left|\rho_{j}(n)\right|^{2} e^{-\pi k_{j}}\left|\Gamma\left(s-\frac{1}{2}-i k_{j}\right)\right|^{2} \\
=\frac{2^{2-2 \sigma}}{\pi} \Gamma(2 \sigma-1) t+o(t),
\end{gathered}
$$

since $\sqrt{f(t)}=o(t)$.

Taking integrals on both sides of (43) yields

$$
\begin{aligned}
& \int_{t-f(t)}^{t+f(t)} \sum_{\left|k_{j}-r\right|<\sqrt{f(r)}}\left|\rho_{j}(n)\right|^{2} e^{-\pi k_{j}}\left|\Gamma\left(\sigma-\frac{1}{2}+i\left(r-k_{j}\right)\right)\right|^{2} d r \\
& =\frac{2^{3-2 \sigma}}{\pi} \Gamma(2 \sigma-1) t f(t)+o(t f(t)) .
\end{aligned}
$$

Interchanging the order of summation and integral in (44), the lefthand side of (44) becomes

$$
\begin{array}{r}
\sum_{\left|k_{j}-t\right|<f(t)}\left|\rho_{j}(n)\right|^{2} e^{-\pi k_{j}} \int_{k_{j}-\sqrt{f(t)}}^{k_{j}+\sqrt{f(t)}}\left|\Gamma\left(\sigma-\frac{1}{2}+i\left(r-k_{j}\right)\right)\right|^{2} d r \\
+o(t f(t)),
\end{array}
$$


by using (37). Note further that

$$
\begin{aligned}
\int_{k_{j}-\sqrt{f(t)}}^{k_{j}+\sqrt{f(t)}}\left|\Gamma\left(\sigma-\frac{1}{2}+i\left(r-k_{j}\right)\right)\right|^{2} d r \\
\quad=\int_{-\infty}^{\infty}\left|\Gamma\left(\sigma-\frac{1}{2}+i r\right)\right|^{2} d r+O\left(e^{-\pi \sqrt{f(t)}}\right) .
\end{aligned}
$$

From this and (44) and (45) it follows that

$$
\begin{aligned}
\sum_{\left|k_{j}-t\right|<f(t)}\left|\rho_{j}(n)\right|^{2} e^{-\pi k_{j}} \\
\quad \sim \frac{2^{3-2 \sigma}}{\pi}\left(\int_{-\infty}^{\infty}\left|\Gamma\left(\sigma-\frac{1}{2}+i r\right)\right|^{2} d r\right)^{-1} \Gamma(2 \sigma-1) \operatorname{tf}(t)
\end{aligned}
$$

for $\sigma=\frac{3}{4}+1 / \log n$ and $n^{1+\varepsilon} \ll t$.

Now if we fix $n$, then we see from the proof that (46) holds good uniformly for $\sigma$ in an interval $I \subset\left(\frac{3}{4}, \infty\right)$. By analytic continuation, there is a constant $\xi$ for which

(47) $\xi \int_{-\infty}^{\infty} \Gamma\left(\sigma-\frac{1}{2}+i r\right) \Gamma\left(\sigma-\frac{1}{2}-i r\right) d r=2^{2-2 \sigma} \Gamma(2 \sigma-1)$

Indeed $\xi=\frac{1}{\pi}$, since

$$
\int_{-\infty}^{\infty}\left|\Gamma\left(\frac{1}{2}+i r\right)\right|^{2} d r=\int_{-\infty}^{\infty} \frac{\pi}{c h \pi r} d r=\pi
$$

This completes the proof of Theorem 1, and equation (47) gives the proof of Theorem 4 .

Finally we prove Theorem 3. For $Y \geq 10, Y \leq x \leq e Y$, and $Y^{2 / 3} \leq T \leq \frac{1}{2} Y$, Lemma 2 gives

$$
\sum_{c \leq x} \frac{S(m, n ; c)}{c}=\sum_{\left|k_{j}\right|<T} \xi_{j} \frac{x^{2 i k_{j}}}{2 i k_{j}}+o(1) .
$$


On applying Lemma 3 to (48), we get

$$
\begin{aligned}
\int_{Y}^{e Y} & \left(\sum_{c \leq x} \frac{S(m, n ; c)}{c}\right)^{2} \frac{d x}{x} \\
& \ll \int_{Y}^{e Y}\left|\sum_{\left|k_{j}\right|<T} \frac{\xi_{j}}{2 k_{j}} x^{2 i k_{j}}\right|^{2} \frac{d x}{x}+o(1) \\
& =\int_{\log Y}^{1+\log Y \mid}\left|\sum_{\left|k_{j}\right|<T} \frac{\xi_{j}}{2 k_{j}} e^{2 i k_{j} u}\right|^{2} d u+o(1) \\
& \ll \int_{-T-1}^{T+1}\left|\sum_{t<k_{j}<t+1} \frac{\xi_{j}}{2 k_{j}}\right|^{2} d t+o(1) \\
& \ll \int_{1}^{T+1}\left(\sum_{\left|k_{j}-t\right|<1} \frac{\left|\rho_{j}(n) \rho_{j}(m)\right|}{c h \pi k_{j}} k_{j}^{-3 / 2}\right)^{2} d t+o(1), \quad \text { by }(29), \\
& \ll \int_{1}^{T+1} \int_{t^{-1} d t+o(1) \quad \text { by Theorem } 1,}^{\ll} \log T \\
& \ll, n \\
& \ll \log _{m} \log Y .
\end{aligned}
$$

This completes the proof of Theorem 3 .

\section{REFERENCES}

[1] P. X. Gallagher, A large sieve density estimate near $\sigma=1$, Invent. Math., 11, (1970), 329-339.

[2] _ Some consequences of the Riemann hypothesis, Acta Arith., 37 (1980), 339-343.

[3] D. Goldfeld and P. Sarnak, Sums of Kloosterman sums, Invent. Math., 71 (1983), 243-250.

[4] N. Y. Kuznietsov, Petersson's conjecture for cusp forms of weight zero and Linnik's conjecture. Sums of Kloosterman sums, Math. USSR Sbornik, 39 (1981)? No. 3, 299-342.

[5] A. Selberg, On the estimation of Fourier coefficients of modular forms, Proc. Sympos. Pure Math., vol. 8, Amer. Math. Soc., Providence, R. I., 1965, pp. $1-15$.

[6] E. C. Titchmarsh, The Theory of the Riemann Zeta Function, Oxford University Press, 1951. 
[7] A. V. Venkov, Spectral Theory of Automorphic Functions, Proceedings of the Steklov Institute of Mathematics 1982, Issue 4.

Received September 29, 1988 and in revised form September 10, 1989.

INSTITUTE OF MATHEMATICS

ACADEMIa Sinica

TAIPEI, TAIWAN 11529 



\section{PACIFIC JOURNAL OF MATHEMATICS EDITORS}

\author{
V. S. VARADARAJAN \\ (Managing Editor) \\ University of California \\ Los Angeles, CA 90024-1555-05 \\ Herbert Clemens \\ University of Utah \\ Salt Lake City, UT 84112 \\ THOMAS ENRIGHT \\ University of California, San Diego \\ La Jolla, CA 92093
}

R. FINN

Stanford University

Stanford, CA 94305

HeRmann FlaschKa

University of Arizona

Tucson, AZ 85721

VAUghan F. R. Jones

University of California

Berkeley, CA 94720

SteVEN KeRCKHOFF

Stanford University

Stanford, CA 94305

\section{C. MOORE}

University of California

Berkeley, CA 94720

MARTIN SCHARLEMANN

University of California

Santa Barbara, CA 93106

HAROLD STARK

University of California, San Diego

La Jolla, CA 92093

\section{ASSOCIATE EDITORS \\ R. ARENS \\ E. F. BECKENBACH \\ B. H. NeUmanN \\ F. WoLF \\ (1904-1989) \\ K. Yoshida (1906-1982)}

\section{SUPPORTING INSTITUTIONS}

UNIVERSITY OF ARIZONA

UNIVERSITY OF BRITISH COLUMBIA

CALIFORNIA INSTITUTE OF TECHNOLOGY

UNIVERSITY OF CALIFORNIA

MONTANA STATE UNIVERSITY

UNIVERSITY OF NEVADA, RENO

NEW MEXICO STATE UNIVERSITY

OREGON STATE UNIVERSITY
UNIVERSITY OF OREGON

UNIVERSITY OF SOUTHERN CALIFORNIA

STANFORD UNIVERSITY

UNIVERSITY OF HAWAII

UNIVERSITY OF TOKYO

UNIVERSITY OF UTAH

WASHINGTON STATE UNIVERSITY

UNIVERSITY OF WASHINGTON

The Supporting Institutions listed above contribute to the cost of publication of this Journal, but they are not owners or publishers and have no responsibility for its content or policies.

Mathematical papers intended for publication in the Pacific Journal of Mathematics should be in typed form or offset-reproduced (not dittoed), double spaced with large margins. Please do not use built up fractions in the text of the manuscript. However, you may use them in the displayed equations. Underline Greek letters in red, German in green, and script in blue. The first paragraph must be capable of being used separately as a synopsis of the entire paper. In particular it should contain no bibliographic references. Please propose a heading for the odd numbered pages of less than 35 characters. Manuscripts, in triplicate, may be sent to any one of the editors. Please classify according to the 1991 Mathematics Subject Classification scheme which can be found in the December index volumes of Mathematical Reviews. Supply name and address of author to whom proofs should be sent. All other communications should be addressed to the managing editor, or Elaine Barth, University of California, Los Angeles, California 90024-1555-05.

There are page-charges associated with articles appearing in the Pacific Journal of Mathematics. These charges are expected to be paid by the author's University, Government Agency or Company. If the author or authors do not have access to such Institutional support these charges are waived. Single authors will receive 50 free reprints; joint authors will receive a total of 100 free reprints. Additional copies may be obtained at cost in multiples of 50 .

The Pacific Journal of Mathematics (ISSN 0030-8730) is published monthly except for July and August. Regular subscription rate: $\$ 190.00$ a year (10 issues). Special rate: $\$ 95.00$ a year to individual members of supporting institutions.

Subscriptions, orders for numbers issued in the last three calendar years, and changes of address should be sent to Pacific Journal of Mathematics, P.O. Box 969, Carmel Valley, CA 93924, U.S.A. Old back numbers obtainable from Kraus Periodicals Co., Route 100, Millwood, NY 10546.

The Pacific Journal of Mathematics at P.O. Box 969, Carmel Valley, CA 93924 (ISSN 0030-8730) is published monthly except for July and August. Second-class postage paid at Carmel Valley, California 93924, and additional mailing offices. Postmaster: send address changes to Pacific Journal of Mathématics, P.O. Box 969, Carmel Valley, CA 93924.

PUBLISHED BY PACIFIC JOURNAL OF MATHEMATICS, A NON-PROFIT CORPORATION Copyright (C) 1991 by Pacific Journal of Mathematics 


\section{Pacific Journal of Mathematics}

Vol. 148, No. $2 \quad$ April, 1991

Yuri A. Abramovich, Operators preserving disjointness on rearrangement

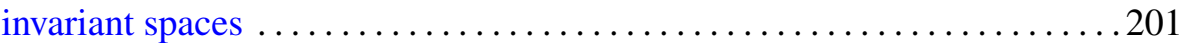

Andrew French Acker and Kirk Lancaster, Existence and geometry of a free boundary problem for the heat equation .................. 207

So-Chin Chen, Real analytic regularity of the Szegő projection on circular

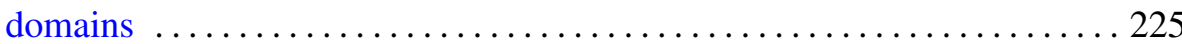

Chen-Lian Chuang, An independence property of central polynomials . . . 237

Peter Larkin Duren and M. Schiffer, Robin functions and energy

functionals of multiply connected domains $\ldots \ldots \ldots \ldots \ldots \ldots \ldots \ldots . \ldots 251$

Johan Henricus Bernardus Kemperman, Sets of uniqueness and systems

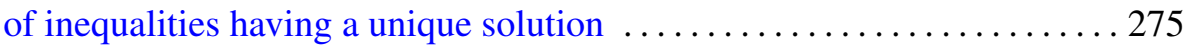

Ka-Lam Kueh, Fourier coefficients of nonholomorphic modular forms and sums of Kloosterman sums . .......................... 303

Gerard J. Murphy, Ordered groups and crossed products of $C^{*}$-algebras . . 319

You-Qiang Wang, The $p$-parts of Brauer character degrees in $p$-solvable

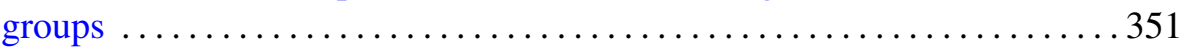

Hidenobu Yoshida, Harmonic majorization of a subharmonic function on a cone or on a cylinder ........................................ 369 